\title{
CO-CONSTRUCTION DE LA CROYANCE DANS LE RÉCIT / DRAME INTERACTIF
}

\author{
Nicolas Szilas ${ }^{1}$
}

Cet article traite de la co-construction de la croyance mise en jeu par le récit interactif, dans lequel l'utilisateur exerce une influence sur le cours de l'histoire. Les questions de répartition des pouvoirs dans ce type d'écriture et de lecture seront discutées en détail, selon le type d'œuvre envisagée.

Nous nous intéressons au cas du récit interactif, que nous définirons, dans le cadre de cet article, comme un récit de fiction médiatisé par un système informatique dans lequel l'utilisateur exerce une influence sur le cours de l'histoire. L'influence de l'utilisateur, sa participation physique dans le « signal narratif » qui lui est finalement transmis, met immédiatement à mal un modèle purement transmissif de la croyance, dans lequel un certain nombre de croyances de l'auteur sont transmises au destinataire du récit. À travers une analyse des mécanismes en jeu dans des récits fortement interactifs, nous montrerons cependant que les mécanismes de co-construction des croyances sont loin de priver l'auteur de son rôle principal. Par une écriture plus abstraite, une écriture de règles, l'auteur met en scène la participation de l'utilisateur et donc reste le plus souvent le « maitre du jeu », quant aux croyances mises en œuvre dans le récit.

1 Nicolas Szilas est Maître d'enseignement et de recherche au TECFA, FPSE à l'Université de Genève (Suisse).

Recherches en communication, $\mathrm{n}^{\circ} 38$ (2012). 


\section{Récit interactif et croyance}

Avant de traiter la question la co-construction de la croyance, il nous faut situer le concept de « croyance » dans le cadre du récit en général, qu'il soit interactif ou non.

Dans une approche pragmatique du récit, c'est-à-dire en considérant le récit dans son contexte d'énonciation, celui-ci est une forme de discours, émis par un auteur, en destination d'un certain public, visant avant toute chose à transmettre une certaine vision du monde, de la vie, de la condition humaine (Adam, 1994). Ainsi, un récit tente, avec plus ou moins d'ostentation, plus ou moins de volonté, de convaincre, au même titre qu'un discours purement argumentatif, mais en usant de caractéristiques qui lui sont propres.

Le propos sur lequel porte le récit, objet de la tentative de persuasion, nous l'appelons ici "croyance ", pour deux raisons. D'une part, ce propos est émis par une personne singulière, l'auteur, dont le rôle est précisément d'apporter sa vision, son traitement, sous forme d'histoire racontée. Le propos du récit est donc éminemment subjectif, et se distingue ainsi du concept de connaissance, qui dans son acceptation scientifique, requiert une validation par plusieurs individus. Le récit, certes, peut être utilisé pour transmettre des connaissances, et il le fera de manière particulièrement persuasive, avec tout l'arsenal qu'on lui connaît : personnages fictifs en proie à toutes sortes de conflits et difficultés, suspense, émotions, empathie, etc. Mais ce faisant, le récit ne change en rien la véracité du propos, uniquement l'impact sur son destinataire. Et l'esprit critique saura prendre distance avec le récit pour reconnaître le contexte narratif et replacer la connaissance en tant que croyance. À la limite, le récit qui transmet une connaissance prend le risque de décrédibiliser le propos universellement admis. D'autre part, le propos du récit concerne l'humain - c'est là une caractéristique fondamentale du récit (Ryan, 2004 ; Adam, 1994). Plus précisément, le récit transmet un système de valeurs humaines (Jouve, 2001), en faisant jouer les personnages autour de ces valeurs. On est en présence de notions à la fois complexes et impalpables, qui invitent à parler de croyance plus que de connaissance.

On notera, pour clore la question des termes et définitions, que les sciences cognitives et l'Intelligence Artificielle utilisent le terme 
de " croyance » pour qualifier ce qui est stocké dans le système de traitement d'un agent. Le terme de connaissance, pourtant très utilisé en informatique, est alors évité pour mettre l'accent sur le fait que cette connaissance est relative à l'agent qui la détient. À ce propos, le terme " croyance » est une traduction du terme belief, et il y a peutêtre là un glissement de traduction, puisque belief se traduirait plus par « conviction » ou « opinion ».

Le récit fictionnel traditionnel (nous n'aborderons pas ici le récit non fictionnel) transmet ou tente de transmettre une croyance selon un mode très particulier:

- il met en scène un univers fictif dans lequel des personnages évoluent ;

- ces personnages vivent des situations denses, condensées de la vie réelle, mettant en jeu des choix ou dilemmes conflictuels ;

- les événements dans ce monde fictionnel sont agencés de manière à provoquer chez le destinataire une série d'émotions fortes, variées, notamment via un processus d'attraction ou de répulsion envers les personnages (empathie, contre-empathie);

- plus précisément, par un processus d'identification, ou d'empathie, le destinataire vit «par procuration » les événements qui arrivent aux personnages, projette ses anticipations (promenades inférentielles selon U. Eco (Eco, 1979), tout en restant impuissant, dans une passivité sécurisante, vis-à-vis de la destinée de ces personnages (Tan, 1996).

C'est l'ensemble de ces caractéristiques (que nous n'avons pas cherché ici à recenser exhaustivement) qui donne au récit son impact particulier. Autrement dit, le récit est modelé de telle manière à nous convaincre. Une autre manière de considérer l'impact du récit est de voir en lui plus qu'un médium efficace : une manière fondamentale d'encoder l'information chez l'humain. Ainsi, selon Bruner, la « réalité » de l'individu psychologique, c'est-à-dire ses croyances, est construite sous une forme narrative (Bruner, 1991).

Nous avons dressé le tableau général du récit, et de son mode de transmission particulier des croyances depuis un auteur vers son destinataire. Qu'en est-il du récit interactif ? Peut-on encore parler de transmission de connaissance au vu de la participation significative du 
destinataire dans la construction de l'histoire ? Quelle co-construction des croyances se dessinerait alors?

Le récit interactif tel que nous l'entendons regroupe à la fois les hypertextes narratifs (Clément, 1995), les fictions interactives (Montfort, 2002), les jeux vidéos d'aventure, les jeux vidéos de simulations de personnages tels que Les Sims ${ }^{\mathfrak{O} 1}$ et les drames interactifs (Szilas, 1999 ; Mateas \& Stern, 2000). Cette dernière catégorie nous concerne plus particulièrement. Il s'agit d'un champs de recherche relativement nouveau, d'orientation plutôt informatique mais à forte coloration interdisciplinaire, visant à donner au spectateur un rôle de personnage-acteur dans une fiction toujours renouvelée et dépendante des choix du spectateur. Au-delà des slogans arborés par les sociétés de jeux vidéos depuis des années déjà (voir, par exemple, les jeux Blade Runner ${ }^{2}$, Fable $^{3}$, Heavy Rains ${ }^{4}$, etc.), il s'agit de résoudre une épineuse difficulté technique: donner de la liberté à l'utilisateur de créer/choisir des voies dans l'histoire qui se déroule, tout en maintenant un intérêt narratif, dicté par une volonté d'auteur. Techniquement, le drame interactif fait appel à l'Intelligence Artificielle, pour diriger des personnages autonomes (Aylett et al., 2005), planifier un déroulement narratif (Young, 1999 ; Barber \& Kudenko, 2007), générer des actions narratives (Sgouros, 1999 ; Szilas, 2007), estimer un impact sur un utilisateur (Szilas, 2001 ; Cheong \& Young, 2008), etc. Le drame interactif, puisqu'il donne beaucoup de possibilité d'actions au joueur, beaucoup d'influence, en tout cas davantage que les autres genres apparentés, pose le plus ouvertement la question de la co-construction de la croyance dans le récit interactif en général.

Citons trois exemples précurseurs de drames interactifs. Dans Façade $^{5}$ (Stern \& Mateas, 2003), l'utilisateur incarne l'ami d'un couple fêtant leurs 10 ans de mariage. Il a été le témoin de leur rencontre, il risque fort d'être le témoin de leur séparation ! Parviendra-t-il à

1 Les Sims (The Sims) est un jeu vidéo de simulation de vie créé par Will Wright en 2000.

2 Blade Runner est un jeu vidéo d'aventure adapté du film du même titre (Philip K. Dick, 1982) créé par le studio Westwood en 1997.

3 Fable est un jeu vidéo développé par Lionhead Studios en 2004.

4 Heavy Rains ou Heavy Rains: The Origami Killer est un jeu vidéo développé par le studio Quantic Dream en 2010.

5 Façade est une fiction interactive développée par InterActiveStory dès 2005. 
intervenir pour sauver le couple ? Se lancera-t-il dans une entreprise de séduction de l'un ou l'autre? Dans The Balance of Power', l'utilisateur incarne un pays tout entier, les États-Unis. Il dispose d'un grand éventail d'actions politiques pour intervenir dans le monde post 11 septembre 2001, des plus pacifiques aux plus belligérantes, choisissant ses alliés et ses ennemis. Dans Mutiny (Szilas et al., 2003 ; Szilas, 2007), l'action se passe dans un galion du XVII ${ }^{\mathrm{e}}$ siècle, et l'utilisateur incarne un jeune matelot fait prisonnier sur ce bateau, tentant de fomenter une révolte pour prendre le contrôle du vaisseau. Choisira-t-il la violence ou un moyen plus pacifique pour atteindre ses fins ? Que seront les répercussions de ces choix sur ses relations avec les autres occupants du navire, notamment la nièce du Capitaine ? Ces trois exemples, précisons-le, restent des prototype expérimentaux, mais ils préfigurent un nouveau mode narratif que nous allons approfondir dans ce qui suit.

\section{Récit interactif: une passation de pouvoir?}

\section{1. L'écriture procédurale}

Si l'on se positionne du côté de l'écriture, le drame interactif amène une perte de contrôle de la part de l'auteur. Selon Louchart et al. (2008, p. 227), " the responsibilities of narratorship become shared between system and interactor $»$ (La responsabilité de narration devient partagée entre le système et l'interacteur). En effet, écrire un drame interactif, c'est renoncer à maîtriser l'expérience, à la fois au niveau d'un événement narratif (par exemple, on ne maîtrisera pas les choix narratifs de l'utilisateur, ses choix de points de vue, certaines variations aléatoires), et surtout au niveau des enchaînements d'action, dynamiquement recalculés pour y incorporer les choix narratifs de l'utilisateur. De plus, pour autoriser cette combinatoire importante, le " scénario " écrit par un auteur relève d'une certaine abstraction (Szilas et al., 2003) qui l'éloigne du concret parfaitement maîtrisable du texte écrit. À ce titre, l'écriture interactive se rapproche de l'écriture théâtrale, dans laquelle ni le jeu d'acteur particulier, ni la réception de

1 Crawford, C. (2010). The Balance of Power. Disponible à : http://www.storytron. com.

2 Mutiny est un jeu créé en 2003 par Kevin Wilson. 
la pièce lors de la représentation ne sont maîtrisés lors de l'écriture proprement dite.

Moins de maîtrise de la part de l'auteur, plus de contrôle de la part de l'utilisateur: l'équation du récit interactif se résume-t-elle ainsi ? Un transfert de pouvoir de l'auteur du récit vers son destinataire ? Certains auteurs, en effet, ont vu dans la littérature numérique en général un renversement des rôles : l'auteur n'est plus l'auteur, le lecteur devient auteur (Landow, 1997).

Pourtant, même dans une œuvre extrêmement ouverte comme Le Livre des Morts ${ }^{1}$, qui donne au lecteur la possibilité d'écrire du texte libre au sein de l'œuvre conçue par l'auteur, ce dernier ne ressent pas cette « perte de pouvoir».

Nous allons montrer que la perte de contrôle de l'auteur sur l'expérience particulière du destinataire et l'augmentation de l'influence de ce destinataire sur cette même expérience ne signifient en rien une perte de pouvoir persuasif de l'auteur. En effet, dans le drame interactif, la quantité d'énoncé qui peut être transmis au destinataire est décuplée, car ce n'est plus une histoire unique qui est transmise mais une multitude d'histoires possibles, potentielles, dont certaines seront actualisées par l'utilisateur. Par exemple, sur un système de récit interactif s'appuyant sur un petit ensemble de fragments ordonnés partiellement, Weyhrauch (1997) montre qu'il y a plus de deux milliards d'ordonnancements possibles. Dans l'une des histoires proposées par IDtension, l'utilisateur avait en moyenne 93 choix possibles, ce qui laisse entrevoir une explosion combinatoire du nombre de possibilités d'histoires (Szilas, 2007). Ainsi, pour transmettre une croyance, l'auteur de récit interactif s'y prend différemment. Plutôt que de choisir une histoire, il en fournit des milliers, des millions, toutes possibles, à charge de l'utilisateur d'en actualiser certaines. Son pouvoir d'influence, vu sous cet angle, est énorme.

Écrit-il ces millions d'histoires possibles ? Bien évidemment non, ce sont les capacités génératives de la technologie employée qui se chargeront de générer ces possibles. Ce qu'écrit l'auteur, ce sont les

1 Malbreil, X. (2010). Le Livre des Morts. Disponible à : http://www.livresdesmorts. com. 
règles de déclenchement de tel ou tel événement narratif. Cette écriture des règles, ou écriture des process correspond à la nature calculatoire, procédurale de l'ordinateur, qui est avant tout un moteur de traitement d'information plus qu'une base de données (Murray, 1997). Comment transmet-on une croyance en écrivant des règles de calcul ? Prenons l'exemple du jeu politique September $12 t h^{1}$. Un village afghan est vu d'un avion. L'utilisateur, au moyen d'une cible, peut depuis l'appareil tirer sur les terroristes qui marchent dans le village. Quand un terroriste est tué, certains dommages collatéraux sont inévitables : des villageois civils meurent aussi. Les villageois pleurent autour des corps, puis certains se transforment en terroristes. Plus la partie avance, plus le joueur peine à contenir tous les terroristes, qui remplissent le village. Dans cet exemple simple, la croyance transmise pourrait s'énoncer ainsi: "Cela ne sert à rien de tuer aveuglément les terroristes, cela ne fait qu'augmenter leur nombre au final ». Dans le programme informatique, elle s'écrit sous la forme de plusieurs règles simples, que nous pouvons spéculer sous cette forme: « Si un proche d'un villageois est tué par l'action du jour, alors il y a $20 \%$ de chances que ce villageois se transforme en terroriste ». C'est bien au travers de l'écriture de ces règles que s'exprime l'auteur. On constate alors que le contrôle que ce dernier perd au niveau de l'enchaînement précis des événements narratifs n'est pas perdu mais déplacé, vers les règles elles-mêmes qui contrôlent la génération des événements.

Avant d'aborder la question de la co-construction de la croyance dans ce contexte, nous sommes maintenant à même de définir ce qui caractérise le drame interactif, par rapport aux autres modes narratifs.

\section{2. Redéfinir le drame interactif}

On distingue traditionnellement deux modes de récit: le mode diégétique (diegesis) et le mode mimétique (mimesis) (Aristote, 1990). Le premier consiste à relater, par le langage écrit ou oral, les événements qui constituent l'histoire. Le roman écrit, l'histoire contée, la nouvelle enregistrée sur cassette ou en podcast relèvent du mode diégétique. Dans ce mode, l'auteur fait imaginer au destinataire (lecteur, auditeur) le monde fictionnel et les événements qui s'y déroulent. Le deuxième

1 Frasca, G. (2010). September 12th. Disponible à : http://www.newsgaming.com/ games/index $12 . \mathrm{htm}$. 
mode consiste à montrer directement les événements de l'histoire, très souvent visuellement, mais aussi auditivement (les autres modalités sensorielles étant difficilement exploitables). Le théâtre, le film, le dessin animé, la bande dessinée, le théâtre radiophonique, etc. relèvent du mode mimétique. Dans celui-ci, l'auteur fait voir/entendre au destinataire le monde fictionnel. On utilise aussi le terme de " drame » (drama en anglais et latin) pour désigner ce mode narratif, s'écartant de sa signification « tragique ».

Qu'en est-il du drame interactif? Est-ce, comme son nom semble l'indiquer, une variante du récit dramatique? Si l'on se place de son point de vue, le destinataire imagine dans le cas du mode diégétique, et voit/entend dans le cas du mode mimétique. Ce qui nous semble fondamentalement différent dans le cas du drame interactif est le fait que le destinataire puisse non plus seulement imaginer et percevoir le monde fictionnel, mais aussi vivre ce monde. En effet, choisir pour un personnage les actions qu'il va effectuer, en observer les conséquences, et ainsi engendrer un monde possible unique (dû à la combinatoire, ce monde est effectivement unique pour chaque séquence d'interaction), constitue une situation de vie, dans un monde fictionnel, virtuel. Contrairement aux deux autres modes, dans lesquels le destinataire est amené à imaginer donc à constituer divers mondes possibles lors du récit (Ryan, 2001), le monde fictionnel du drame interactif, dans lequel le destinataire vit, sort de l'imagination et se matérialise dans l'artefact choisi, généralement par un système informatique.

De point de vue de l'auteur, ce nouveau mode lui permet de faire vivre ses lecteurs/auditeurs/spectateurs. Nous proposons donc de le nommer le mode "démiurgique », en référence au grec dêmiourgos, qui signifie le créateur de l'univers. C'est en effet un véritable univers qui est créé, virtuel dans sa matérialisation, mais bien réel dans sa capacité à faire agir en tant que personnage, donc de faire vivre un utilisateur. Certes, dans la création fictionnelle classique, l'auteur est déjà en position démiurgique, créant l'univers dans lequel évoluent ses personnages. Ainsi, Souriau (1950, p. 166) écrit: « La connaissance des cadres combinatoires nécessaires, opératoirement contraignant [...] fait de l'imagination inventive [...] la démiurgie instauratrice elle-même, créant ou faisant à sa volonté les situations, actes illuminatifs du cosmos théâtral. » Mais les situations mentionnées par Souriau finissent couchées sur le papier, linéarisées, alors que les situations du drame 
interactif sont l'objet d'une action de l'utilisateur en tant que partie prenante de cette situation via le personnage qu'il incarne.

\section{3. Co-construction de la croyance dans le mode démiurgique}

L'écriture procédurale mentionnée ci-dessus, qui consiste à écrire des règles pour engendrer (générer) des possibles, est simplement un moyen technique pour parvenir à la diversité requise par la participation importante de l'utilisateur. La nature démiurgique du récit interactif apporte un éclairage complémentaire sur la transformation radicale du rôle de l'auteur dans ces récits. S'il écrit des règles, c'est pour faire vivre "son petit monde ", à savoir les personnages non joueurs, le monde physique virtuel et l'utilisateur. Créateur d'univers (le terme est d'ailleurs utilisé dans le monde du jeu vidéo), il en maîtrise les règles et tente d'en maîtriser le déroulement. Pour ainsi dire, il se prend pour Dieu.

Dans ce contexte, on demeure dans une situation où la croyance de l'auteur est transmise vers les destinataires de l'œuvre. Certes, on n'a ni une transmission directe (assertion d'un fait), ni une transmission narrativisée (transmission de valeurs via un récit), mais une transmission procédurale, qui consiste à spécifier des règles et laisser le destinataire jouer avec ces règles pour en découvrir la croyance associée. La co-construction de la croyance est donc limitée, puisque l'utilisateur ne peut s'exprimer uniquement dans le cadre qui lui est proposé. La participation de ce dernier n'est pas vaine pour autant. En exerçant son action sur le système de règles écrites par l'auteur, il en éprouve la validité, recherche des chemins possibles, explore une partie des possibles sous-jacents. La croyance transmise n'en est que renforcée, et ce pour trois raisons. Premièrement, ce n'est pas une histoire particulière qui est donnée, dont on pourra toujours reprocher son côté précisément particulier, singulier, exceptionnel, mais un faisceau d'histoires, ce qui renforce le message. Qui plus est, ces histoires ne sont pas données au destinataire - il y en existerait des milliers voire des millions -, mais suscitées par les choix de l'utilisateur. L'utilisateur constate donc que, quoi qu'il fasse, l'histoire générée va dans le sens de la croyance émise par l'auteur. Deuxièmement selon un principe constructiviste d'apprentissage, la croyance est construite par l'action directe sur l'environnement, pas uniquement par une action en pensée, ce qui laisse supposer un impact plus fort. Troisièmement, cette exploration des possibles est associée à un certain plaisir, certainement proche d'un 
plaisir ludique, dont on peut supposer qu'il facilite la réception de la croyance.

Pour synthétiser, on pourra dire que le drame interactif, tel qu'il est aujourd'hui envisagé, s'il autorise une co-construction de l'histoire, participe moins à une co-construction des croyances, puisque la co-construction de l'histoire pendant la lecture s'effectue selon des croyances stables spécifiées par l'auteur. Ce contraste, qui résulte du passage d'une écriture centrée sur les événements vers une écriture centrée sur les règles, est donc une configuration de communication bien singulière que nous allons analyser davantage dans les deux sections suivantes.

\section{Manipulation du drame interactif?}

$\mathrm{Si}$, d'un côté, le drame interactif permet à un auteur de donner la parole à son lecteur en lui laissant la possibilité d'influer fortement sur le déroulement de l'histoire et si, de l'autre, il s'assure que le propos, les valeurs, l'issue de l'œuvre restent maîtrisés par le système informatique, n'est-on pas face à une forme de manipulation particulièrement pernicieuse ? En effet, on serait dans une position de «laisser croire » à une liberté de manœuvre, alors que « les dés sont pipés », le contrôle reste dans les mains de l'auteur.

Par exemple, dans le jeu de simulation Energyville ${ }^{1}$, le joueur est amené à effectuer un certain nombre de grands choix énergétiques, sur une durée de 30 ans. Le but du jeu est de montrer que les choix énergétiques ne sont pas simples, et que, par exemple, une solution « toute éolienne » n'est pas satisfaisante à long terme. Le jeu permet en effet à l'utilisateur de faire l'expérience de certains choix stratégiques, apparemment loin de toute croyance directement transmise par un auteur. Pourtant, connaissant le commanditaire du jeu, la société pétrolière américaine Chevron, on pourra certainement relever les inévitables biais de la simulation sous-jacente au jeu, et dénoncer la manipulation de ce produit. Le « on » ci-avant désignera préférentiellement le militant « anti-multinationales pétrolières », utilisateur averti, qui à son tour pourra conseiller un autre jeu en ligne, Oiligarchy ${ }^{2}$, jeu politique

1 Chevron. (2010). Energyville. Disponible à : http://www.willyoujoinus.com/ energyville.

2 Molleindustria. (2010). Oiligarchy. Disponible à : http://www.addictinggames.com/ 
dénonçant les liens pétrole-pouvoir politique, jeu qui à son tour pourra être dénoncé pour la fausse liberté qu'il offre.

Sans être déjà au courant des problématiques concernées par le jeu, l'utilisateur naïf sera donc manipulé. Cette manipulation est par exemple dénoncée dans un ouvrage consacré au storytelling (Salomon, 2007). Mais il faut relativiser à plusieurs égards la manipulation pernicieuse du drame interactif.

D'une part, il faut se rappeler que le récit lui-même est déjà une manipulation, car, nous l'avons vu plus haut, il transmet des croyances de manière indirecte mais efficace. Le lecteur/spectateur/auditeur actuel en a relativement conscience et sait ne pas être d'accord avec tel récit. Dans le domaine interactif, le contrat de lecture est encore balbutiant, mais, en gagnant en maturité, le drame interactif proposera un contrat de lecture dans lequel la subjectivité procédurale sera connue des utilisateurs et acceptée par ceux-ci, en même temps qu'elle sera maîtrisée et assumée par l'auteur.

D'autre part, tout démiurge que le drame interactif demande implicitement de devenir, l'auteur n'en demeure pas moins humain et limité. Le système de règles qu'il conçoit pour convaincre est rapidement complexe à gérer, et dépasse ses capacités de prévision. Il n'est pas rare que le système créé, dès qu'il atteint un certain niveau de complexité, puisse échapper aux prédictions de ses auteurs, non qu'il soit mal conçu et qu'il produise des situations erronées, mais parce qu'il produit une situation simplement surprenante et non anticipée. Ainsi, dans le jeu Les Sims ${ }^{\mathcal{O}}$, un jeu qui certes ne raconte pas une histoire mais en ce qu'il met en scène des personnages en interaction constitue tout de même un "proto-récit », on peut supposer que l'auteur n'avait pas prévu que certains joueurs allaient enfermer une grand-mère virtuelle entre quatre murs d'un mètre, et l'observer mourir ; que d'autres allaient créer des environnements oniriques dans lesquels ils allaient se mettre en scène pour produire une œuvre littéraire, comme dans le livre Corpus Simsi ${ }^{1}$; etc. Ainsi, si ce jeu met effectivement en scène une société de consommation matérialiste et sur cette thématique transmet une croyance avec efficacité, cohérence et solidité, il se « fissure » en

oiligarchy.html.

1 Delaume, C. (2003). Corpus Simsi. s. 1. : Éditions Léo Scheer. 
d'autres points où d'autres croyances viennent à émerger, d'abord chez un utilisateur particulier, puis de manière amplifiée via les réseaux sociaux constitués sur Internet. Il ne s'agit pas à ce niveau de pointer un quelconque défaut du jeu ; bien au contraire, ce type de phénomène, qui d'ailleurs porte un nom : le emergent gameplay est recherché par les concepteurs et les joueurs. Il s'agit par contre de pointer que, dans le cadre du mode démiurgique, il semble que le message ne puisse finalement pas être totalement maîtrisé, car la complexité du système dynamique engendré par les règles qui le régissent engendre facilement des situations inattendues, qui amènent l'utilisateur à construire une croyance qui se détache de celle de l'auteur. On objectera que, dans une œuvre narrative linéaire, il y a déjà place à interprétation, donc également à une co-construction de croyances. Mais ce qui distingue le récit interactif, c'est que l'on n'a pas à faire à une interprétation seulement mentale. La co-construction de la croyance par l'utilisateur s'établit avec le même dispositif narratif que celui utilisé par l'auteur.

Ainsi, pour résumer, le drame interactif se présente comme un médium ouvert, mais contrôlé strictement par des règles écrites par l'auteur qui, finalement, peuvent laisser la place au détournement par l'utilisateur. En tant qu'auteur, choisir le récit interactif pour émettre un croyance, c'est donc se lancer dans une écriture avec une double inconnue : d'une part, l'histoire engendrée par chaque lecteur et même par chaque lecture est différente - par définition-; d'autre part, ce lecteur pourrait trouver dans l'œuvre un terrain de construction d'une croyance différente. Bien-sûr, nous parlons ici d'œuvres fortement interactives, encore pour la plupart en gestation, mais que les recherches actuelles laissent entrevoir dans un futur proche.

\section{Vers un renversement des rôles?}

À l'heure d'Internet, du Web 2.0, et du user generated content, nous souhaiterions aborder le statut de la co-construction des croyances pour des œuvres plus ouvertes, dans lesquelles le destinataire est lui-même auteur à l'image, par exemple, de ces multiples sites de construction de ses propres bandes dessinées. Ce type de récit interactif consisterait à laisser à l'auteur-destinataire le soin d'écrire/sélectionner/paramétrer les règles sous-jacentes d'un récit interactif, lui-même destiné à un destinataire final. Ce type de système n'existe pas aujourd'hui dans le cadre du drame interactif tel que nous l'avons abordé, mais nous nous 
permettons cependant d'ouvrir une parenthèse prospective en imaginant deux familles de systèmes. Dans les premiers systèmes, que nous appellerons " outils auteur », l'auteur-destinataire utiliserait un outil auteur pour lui permettre de construire son propre récit interactif. Ces systèmes existent dans le cadre des jeux vidéos, à travers les éditeurs de niveaux qui permettent, à partir d'un jeu existant, de créer un niveau personnalisé, appelé un « mod». Dans les seconds systèmes, il s'agirait d'exploiter les histoires enchâssées (Ryan, 1991), très fréquentes dans les récits, c'est-à-dire le concept d'histoire dans l'histoire (voir par exemple Les Mille et Une Nuits). À l'intérieur d'un récit interactif, l'utilisateur pourrait avoir suffisamment de liberté pour contrôler les règles d'un sous-récit. Par exemple, il pourrait contrôler un personnage capable de commander d'autres personnages, en leur donnant des instructions précises. Il « comploterait » (noter l'étymologie du mot : « intriguer avec »), à la manière d'un film de cambriolage, ou d'une pièce de Marivaux ou Beaumarchais. Si un tel récit est mono-utilisateur, alors le « comploteur » est lui-même le destinataire de ce sous-récit, mais si le récit est multi-utilisateur, alors le pouvoir de contrôler les règles du récit lui permet à son tour d'être l'auteur d'un sous-système de croyances.

C'est ce type de système, plutôt du premier type, qui est appelé par l'auteur du jeu September 12th discuté plus haut, pour précisément donner plus de pouvoir à l'utilisateur (Frasca, 2001). Inspiré par le Théâtre de l'Opprimé d'Augusto Boal (Boal, 2003), il imagine une version du jeu Les $\operatorname{Sims}^{\mathcal{O}}$ dans laquelle l'utilisateur pourrait créer ou modifier les règles comportementales d'un personnage virtuel, afin de traiter une problématique particulière. Il prend l'exemple d'un personnage alcoolique. Ainsi, ce qu'il appelle les video games of the oppressed permettraient à un utilisateur de mettre en scène ses propres croyances, et de les diffuser à la communauté des joueurs.

Dans cette nouvelle configuration, la co-construction de la croyance est davantage centrée sur l'auteur-destinataire, le premier destinataire du récit interactif, puisqu'il a la possibilité d'écrire les règles d'un récit et donc d'être lui-même le « créateur » dans le mode démiurgique propre au drame interactif. Cependant, cet auteur-destinataire ne peut écrire de règles que dans le cadre imposé par l'auteur initial. Ainsi, l'auteur écrit des méta-règles, des règles décrivant les règles possibles. Ce faisant, il part toujours d'une croyance, qu'il transmet d'une 
manière certes encore plus indirecte, par l'établissement d'un cadre d'écriture, d'autres règles, mais d'autant plus efficaces. La construction des croyances dans ces systèmes futurs sera donc partagée entre trois instances : l'auteur, l'auteur-destinataire et le destinataire final, dans un mode à la fois collaboratif et hiérarchique.

\section{Conclusion}

Nous avons abordé la question de la co-construction de la croyance dans un médium narratif récent, voire à venir, appelé drame interactif. Celui-ci se situe à une position intermédiaire, en matière de participation, entre le récit linéaire, qui guide le destinataire sur un chemin unique, et les formes plus ouvertes dans lesquelles le destinataire est amené à jouer un rôle de créateur de contenu. Nous y avons trouvé des modes de co-construction souvent ambivalents, où se mêlent liberté de participation et contrôle « en règles » par l'auteur.

Les modes de communication de ce médium encore balbutiant sont amenés à la fois à se cristalliser en genres définis, mais aussi à se diversifier vers des œuvres et genres toujours plus complexes, au niveau de la co-construction des croyances. Les contrats de lecture incluront l'ambiguité, l'ironie, le second degré, des postures d'auteur soit esquivées, soit assumées. Le rôle de l'auteur dans la construction des croyances, loin de s'amoindrir, investira des terrains toujours plus riches en termes d'abstraction, d'univers engendrés et de niveaux de lecture. Le lecteur, quant à lui, développera de nouvelles compétences de lecture, plus précisément des compétences d'exploration, de jeu et de prise de recul par rapport aux mécanismes mis en œuvre.

\section{Remerciements}

Ce travail a été financé en partie par la Commission Européenne, sur le contrat IRIS (FP7-ICT-231824). 


\section{Références}

Adam, J.-M. (1994). Le texte Narratif. Paris : Nathan.

Aristote. (1990). La Poétique, introduction, traduction nouvelle et annotation de Michel Magnien. Paris : Livre de poche classique (édition originale : -330).

Aylett, R., Dias, J., Louchart, S., Paiva, A., \& Vala, M. (2005). FearNot! An Experiment in Emergent Narrative. Dans Proceedings of the $5^{\text {th }}$ International Conference on Intelligent Virtual Agents (pp. 305-316). Heidelberg : Springer Verlag.

Aylett, R., Kriegel, M., Louchart, S., \& Swartjes, I. (2008). Purposeful Authoring for Emergent Narrative. Dans U. Spierling \& N. Szilas (Éd.) Proceedings of the First Joint Conference on Interactive Digital Storytelling (ICIDS), Lecture Notes in Computer Science 5334 (pp. 273-284). Heidelberg : Springer Verlag.

Barber, H., \& Kudenko, D. (2007). Dynamic Generation of Dilemma-based Interactive Narratives. Dans Proceedings of the Artificial Intelligence and Interactive Digital Entertainment conferance - AIIDE 2007. Menlo Park, California : AAAI Press.

Boal, A. (2003). Le théâtre de l'opprimé. Paris : La Découverte.

Bruner, J. (1991). The Narrative Construction of Reality. Critical Inquiry, 18 (1), 1-21.

Cheong, Y.G., \& Young, M.R. (2008). Narrative Generation for Suspense: Modeling and Evaluation. Dans U. Spierling \& N. Szilas (Éd.) Proceedings of the First Joint Conference on Interactive Digital Storytelling (ICIDS), Lecture Notes in Computer Science 5334 (pp. 157-158). Heidelberg : Springer Verlag.

Clément, J. (1995). L'hypertexte de fiction : naissance d'un nouveau genre ? Dans A. Vuillemin \& M. Lenoble (Éd.) Littérature et informatique : la littérature générée par ordinateur. ARRAS : Artois Presses Université. Disponible à : http://hypermedia. univ-paris8.fr/jean/articles/allc.htm.

Eco, U. (1979). Lector in Fabula. Milan : Bompiani.

Frasca, G. (2001). Video Games of the Oppressed: Videogames as a Means for Critical Thinking and Debate. Dissertation doctorale, School of Literature, Communication and Culture, Georgia Institute of Technology, Atlanta.

Jouve, V. (2001). Poétique des valeurs. Paris : Presses Universitaires de France.

Landow, G. P. (1997). Hypertext 2.0: The Convergence of Contemporary Literary Theory and Technology. Baltimore : Johns Hopkins University Press.

Mateas M., \& Stern A. (2003). Integrating Plot, Character and Natural Language Processing in the Interactive Drama Façade. Dans Göbel et al. (Éd.) Proceedings TIDSE'03. Darmstadt : Frauenhofer IRB Verlag.

Mateas, M., \& Stern, A. (2000). Towards Integrating Plot and Character for Interactive Drama. Dans Working Notes of the Social Intelligent Agents: The Human in the Loop Symposium, AAAI Fall Symposium Series. Menlo Park : AAAI Press.

Montfort, N. (2002). Toward a Theory of Interactive Fiction. 29 December 2002, Version 3. Disponible à : http://nickm.com/if/toward.html.

Murray, J. (1997). Hamlet on the Holodeck. The Future of Narrative in the Cyberspace. New York : Free Press.

Ryan, M.-L. (1991). Possible Worlds, Artificial Intelligence, and Narrative Theory. Indiana University Press.

Ryan, M.-L. (2001). Beyond Myth and Metaphor. The Case of Narrative in Digital Media. Game Studies. The International Journal of Computer Game Research [en ligne], 1(1), Disponible à: http://www.gamestudies.org/0101/ryan/. 
Ryan, M.-L. (2004). Introduction. Dans M.-L. Ryan (Éd.) Narrative Across Media: The Languages of Storytelling (pp. 1-40). Lincoln : University of Nebraska.

Salomon, C. (2007). Storytelling. La machine à fabriquer des histoires et à formater les esprits. Paris : La Découverte.

Sgouros, N. M. (1999). Dynamic Generation, Management and Resolution of Interactive Plots. Artificial Intelligence, 107(1), 29-62.

Souriau, E. (1950). Les deux cent mille situations dramatiques. Bibliothèque d'esthétique, Paris: Flammarion.

Szilas, N. (1999). Interactive Drama on Computer: Beyond Linear Narrative. Dans Papers from the AAAI Fall Symposium on Narrative Intelligence, Technical Report FS-99-01. (pp. 150-156). Menlo Park : AAAI Press.

Szilas, N. (2001). A New Approach to Interactive Drama: From Intelligent Characters to an Intelligent Virtual Narrator. Dans Proceedings of the Spring Symposium on Artificial Intelligence and Interactive Entertainment (pp. 72-76). Menlo Park : AAAI Press.

Szilas, N. (2007). A Computational Model of an Intelligent Narrator for Interactive Narratives. Applied Artificial Intelligence, 21(8), 753-801.

Tan, E. (1996). Emotion and the Structure of Narrative Film. Film as an Emotion Machine. Mahwah (NJ) : Erlbaum.

Weyhrauch, P. (1997). Guiding Interactive Drama. Dissertation doctorale, School of Computer Sciences, Carnege Meelon University.

Young, R. M. (1999). Notes on the Use of Plan Structure in the Creation of Interactive Plot. Dans Papers from the AAAI Fall Symposium on Narrative Intelligence, Technical Report FS-99-01. (pp. 164-167). Menlo Park : AAAI Press. 